\title{
Impact of an mHealth supported healthy lifestyle intervention on behavioural stage of change in overweight and obese pregnancy
}

\author{
K. Ainscough, M. Kennelly, K.L. Lindsay, E.J. O’Sullivan and F.M. McAuliffe \\ UCD Obstetrics and Gynaecology, School of Medicine and Medical science, University College Dublin, \\ National Maternity Hospital, Dublin, Ireland
}

Overweight and obesity in pregnancy significantly increases the risk of adverse maternal and infant health outcomes ${ }^{(1)}$. Overweight and obese women often report poor motivation and low self-efficacy ${ }^{(2)}$ as barriers to positive health behaviour change. Mobile health (mHealth) technologies offer significant potential to support lifestyle interventions through delivery of behaviour change techniques and remote, consistent engagement with intervention content ${ }^{(3)}$. However, there is a paucity of published studies examining the effectiveness of mHealth applications in pregnancy ${ }^{(4)}$. The aim of this study is to investigate the influence of a smartphone app-supported antenatal healthy lifestyle intervention on behavioural stage of change among overweight and obese pregnant women.

In this randomised controlled trial ${ }^{(5)}$ we recruited and randomised overweight and obese women (body mass index $\geq 25 \mathrm{and}<40 \mathrm{~kg} / \mathrm{m}^{2}$ ) in early pregnancy (mean gestational age $=15$ weeks). The control group $(n=98)$ received routine antenatal care, which currently offers no consistent diet or lifestyle advice. The intervention group $(n=106)$ received a healthy lifestyle package consisting of individualised nutrition counselling and exercise advice, supported by a smartphone app which included a database of healthy recipes, nutrition and exercise tips and motivational messages. Behavioural stage-of-change score $(1-5 ; 1=$ Pre-contemplation, $2=$ Contemplation, $3=$ Preparation, 4 = Action, 5 = Maintenance) was measured at baseline and in late pregnancy (28 weeks) using a questionnaire developed from the Transtheoretical Model, ${ }^{(6)}$ which assesses 'readiness' to engage in new or change existing lifestyle behaviours.

At baseline, there were no significant differences between intervention and control groups for demographic characteristics (data not shown) or distribution across stage of change category $\left(\chi^{2}=6 \cdot 2, P=0 \cdot 19\right)$. From baseline to 28 weeks, a shift in stage-of-change score distribution was observed for both groups (Fig. 1 \& Fig. 2), which was more significant for the intervention group $(P<$ 0.001 versus $P=0.032)$. At 28 weeks, a significantly higher proportion of the intervention group were at stage $5(52.8 \%)$ versus the control group $(32.7 \%),\left(\chi^{2}=8.4, P=0.004\right)$.

Overweight and obese women enter pregnancy prepared to make positive health behaviour changes (stage 2-3, Figure 1). This mHealth-supported behavioural intervention assisted women in transitioning from contemplation/preparation to maintenance stage of healthy lifestyle behaviours in pregnancy (stage 5, Figure 2). If sustained, this change has potential for a positive impact on pregnancy outcome and long-term health behaviours for mother and child.

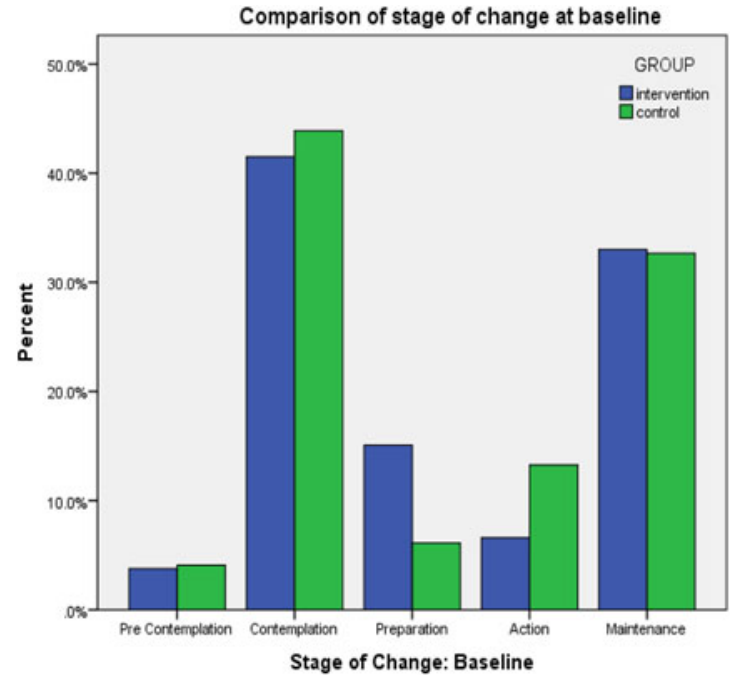

Fig. 1. Comparison of stage of change at baseline.

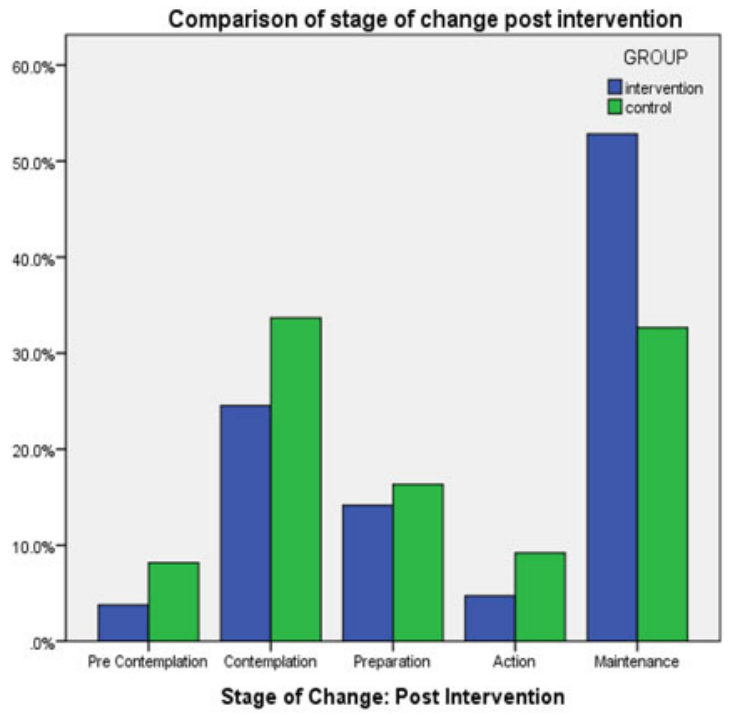

Fig. 2. Comparison of stage of change post intervention.

1. Catalano PM \& Ehrenberg HM (2006) BJOG 113/10, 1126-1133.

2. Sui Z, Turnbull D, Dodd J (2013) AMJ 6/11, 565-577.

3. Lefebvre C (2009) Health Promot Pract 10/4, 490-494.

4. O'Brien OA, McCarthy M, Gibney ER et al. (2014) Eur J Clin Nutr 68, 760-766.

5. Kennelly M, Ainscough KA, Lindsay KL et al. (2015) Contemp Clin Trials 46, 92-99.

6. Prochaska JO \& DiClemente CC (1983) J Consult Clin Psychol 51/3, 390-395. 